\title{
Gaussian Approximation for Sum-Product Decoding of Low-Density Parity-Check Codes
}

\author{
Sae-Young Chung \\ Laboratory for Information and \\ Decision Systems, M.I.T. \\ e-mail: sychungelids.mit.edu
}

\author{
Rüdiger Urbanke \\ Communications Theory Lab, \\ EPFL, Lausanne, Switzerland \\ e-mail: Rudiger.UrbankeCepfl.ch
}

\author{
Thomas J. Richardson \\ Lucent Technologies, \\ Murray Hill, NJ \\ e-mail: tjrolucent.com
}

\begin{abstract}
We use a Gaussian approximation (GA) for analyzing the sum-product algorithm for lowdensity parity-check (LDPC) codes and memoryless binary-input continuous-output additive white Gaussian noise (AWGN) channels. This simplification allows us to calculate the threshold quickly and to understand the behavior of the decoder better. We have also designed high rate LDPC codes using the GA that have thresholds less than $0.05 \mathrm{~dB}$ from the Shannon limit.
\end{abstract}

\section{INTRODUCTION}

For many interesting channels and iterative decoders, LDPC codes exhibit a threshold phenomenon: an arbitrary small bit error probability can be achieved if the noise leve is smaller than a certain threshold and the probability of bit error is larger than a positive constant for a noise level above the threshold as the block length tends to infinity [1].

In this paper, we present a simple method to estimate the thresholds of randomly constructed irregular LDPC codes for memoryless binary-input continuous-output AWGN channels under sum-product decoding. This method is based on approximating densities of log-likelihood ratio (LLR) messages as Gaussian mixtures. We assume for each variable node the graph is a tree up to a certain depth as validated by the general concentration theorem [2].

\section{Gaussian Approximation}

If all incoming messages of a variable node are Gaussian, then the resulting extrinsic information distribution is also Gaussian because it is the sum of independent Gaussian random variables. Numerical results using density evolution (DE) [1] show that the extrinsic information distributions from both variable and check nodes are very close to Gaussian even though the inputs are not. From now on, we assume all extrinsic information distributions are Gaussian. By enforcing the consistency condition [2] at each iteration, we can greatly improve the accuracy of the approximation and reduce the $D E$ problem to a one-dimensional one.

Let $\lambda(x)=\sum_{i=2}^{d_{i}} \lambda_{i} x^{i-1}$ and $\rho(x)=\sum_{i=2}^{d_{r}} \rho_{i} x^{i-1}$ be the degree sequences for the variable and check nodes, respectively. For $0<s<\infty$ and $0 \leq t<\infty$, we define $f(s, t)$ as

$$
f(s, t)=\sum_{j=2}^{d_{r}} \rho_{j} \psi^{-1}\left(\left[\sum_{i=2}^{d_{1}} \lambda_{i} \psi(s+(i-1) t)\right]^{j-1}\right),
$$

where $\psi(x)$ is defined by

$$
\psi(x)= \begin{cases}\frac{1}{\sqrt{4 \pi x}} \int_{R} \tanh \frac{u}{2} e^{-\frac{(u-x)^{2}}{4 x}} d u & \text { if } x>0 \\ 0 & \text { if } x=0 .\end{cases}
$$

The message update rule becomes now $t_{l}=f\left(s, t_{l-1}\right)$, where $s=m_{u_{0}}$ is the mean of $u_{0}$ and $t_{l}$ is the ensemble mean of the output messages of check nodes at $l$-th iteration. The initial value $t_{0}$ is 0 . Note that since $t_{1}=f(s, 0)>0$ for $s>0$, the iteration will always start.

We define the threshold $s^{*}$ as the infimum of all $s$ in $\mathbb{R}^{+}$ such that $t_{l}(s)$ converges to $\infty$ as $l \rightarrow \infty$. By finite induction, we conclude that if $s>s^{*}, t_{l}(s)$ converges to $\infty$. The following lemma shows an alternative interpretation of the threshold.

Lemma $1 t_{l}(s)$ will converge to $\infty$ iff

$$
t<f(s, t), \quad \forall t \in \mathbb{R}^{+} .
$$

As in the case of DE [2] we can derive a stability condition:

Theorem 1 If $\lambda_{2}<\lambda_{2}^{*}$, then $t$ will converge to infinity if the initial value of $t$ is lange enough. If $\lambda_{2}>\lambda_{2}^{*}$, then $t$ cannot converge to infinity for any initial value of $t$, where $\lambda_{2}^{*}=e^{1 / 2 \sigma_{n}^{2}} / \prod_{j=2}^{d_{r}}(j-1)^{\rho_{j}}$.

For this model it is even possible to derive expressions for the convergence rate of the probability of error $P_{l}$. In particular, for $\lambda_{2}<\lambda_{2}^{*}, P_{l}$ behaves asymptotically as the following as $l \rightarrow \infty$ :

$$
P_{l} \approx \frac{a}{\sqrt{b+l}}\left(\frac{\lambda_{2}}{\lambda_{2}^{*}}\right)^{2 l},
$$

where $a$ and $b$ are constants that depend on $\lambda(x), \rho(x)$ and $s$. These predictions fit well with the actual results using $D E$.

III. Optimization of Degree Sequences

For given $\rho(x)$ and rate, we can find optimal $\lambda(x)$ that maximizes the noise threshold. This can be performed by maximizing the rate subject to the normalization and the inequality constraint in (1), which can be done using linear programming. Optimization of $\rho(x)$ can be done similarly. We show when we consider only low error probability regions, the optimal form of $\rho(x)$ is concentrated in 1 or 2 consecutive degrees. We have successfully optimized degree sequences using these methods up to within $0.05 \mathrm{~dB}$ from the Shannon limit for rates greater than 0.99 . Good degree sequences were also obtained for lower rates. Online demonstration of degree sequence optimization using the GA and more results are available at http://truth.mit.edu/ sychung.

\section{ACKNOWLEDGMENTS}

The authors would like to express their appreciation to Prof. G. D. Forney, Jr. for his many helpful comments on this paper.

\section{REFERENCES}

[1] T. J. Richardson and R. Urbanke, "The Capacity of LowDensity Parity Check Codes under Message-Passing Decoding," November 1998, submitted to IEEE IT.

[2] T. J. Richardson, A. Shokrollahi and R. Urbanke, "Design of Provably Good Low-Density Parity Check Codes," April 1999, submitted to IEEE IT. 